\section{BIOTECHNOLOGY \\ Turn it off}

Gene 'silencing' with small interfering RNA (siRNA) is attracting considerable interest at present as a strategy for target validation that also has potential therapeutic applications. Originally discovered as part of a natural mechanism for gene regulation in nematodes, fruitflies and plants, interference of gene expression by siRNA — in which siRNA guides sequence-specific messenger RNA degradation and thus prevents it being translated into protein - has recently been shown to be applicable using designed siRNA in mammalian cells. To achieve in vivo gene silencing in mammalian tissues would require expression from intracellular transcription, a challenge that has been tackled by Davidson and colleagues, who now describe a virusmediated delivery mechanism for siRNA that can be used to diminish expression of exogenous and endogenous genes markedly in vitro and in vivo.

To show proof-of-principle in vitro, human cancer cells that express the enzyme $\beta$-glucuronidase were infected with recombinant adenovirus-based vectors that contained the coding sequence for siRNA against $\beta$-glucuronidase, which led to a $60 \%$ decrease in enzyme activity. The authors then assessed the ability of virally expressed

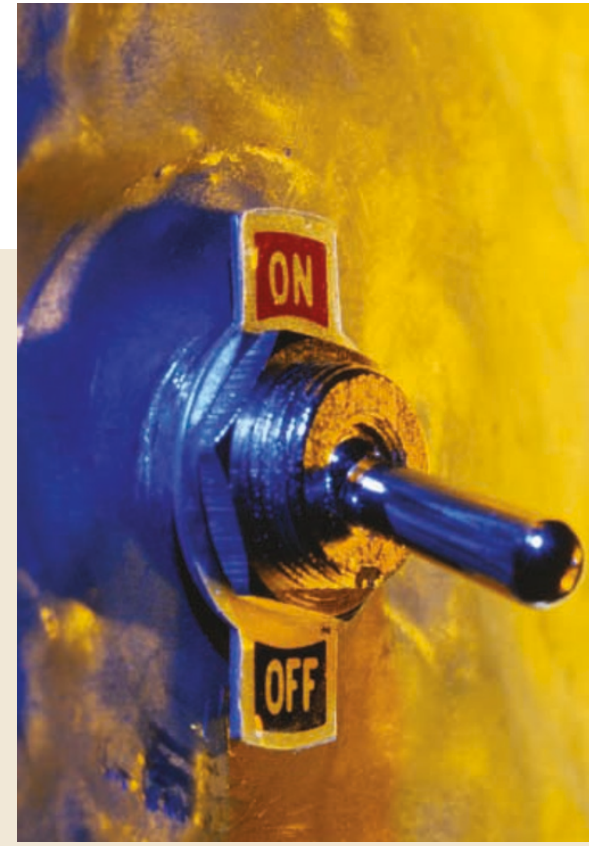

siRNA to diminish target-gene expression in adult mouse tissues in vivo. A significant reduction in the expression of green-

fluorescent protein in the brain of transgenic mice engineered to express this marker protein, and in the endogenous expression of $\beta$-glucuronidase in the liver, was achieved, providing the first demonstration of the use of siRNA to diminish target-gene expression in brain and liver tissue.

One potential therapeutic application of siRNA would be to reduce the expression of toxic gene products associated with disorders such as Huntington's disease, in which the production of a form of a protein that contains an expanded sequence of glutamine residues leads to protein aggregation. The authors showed that expression of a protein containing an expanded polyglutamine sequence in neural cells could be reduced by $90 \%$ using virally expressed siRNA, and this reduction in expression resulted in a significant reduction in the formation of polyglutamine aggregates. Using viral vectors based on adeno-associated viruses and lentiviruses, which have the ability to infect cells in the central nervous system more efficiently than adenovirus vectors, should further extend the application of siRNA to viral-based therapies and target validation.

Peter Kirkpatrick

(10) References and links ORIGINAL RESEARCH PAPER Xia, H. et al. siRNAmediated gene silencing in vitro and in vivo. Nature Biotechnol. 2002 Sep 16 (doi: 10.1038/nbt739)

\title{
Complex is crystal clear
}

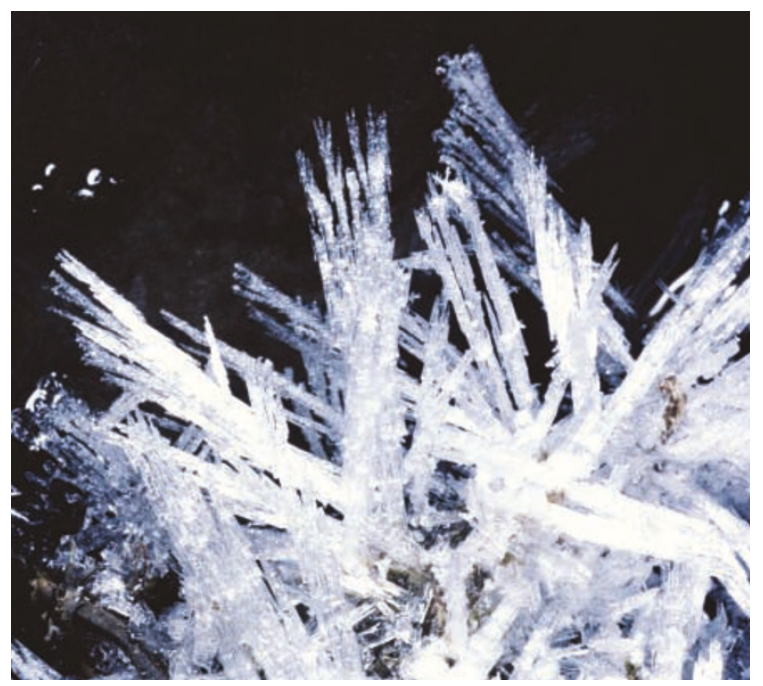

The identification of members of the epidermal growth factor receptor (EGFR) family on cancer cells created a flurry of research to find therapeutic compounds that target the receptors (for example, the monoclonal antibody treatment Herceptin). But the race has also been on to determine the crystal structure of the ligand-receptor complex, to allow new compounds to be discovered on the basis of rational drug design. Now, in Cell, two groups have claimed victory.

Garrett and colleagues looked at the EGFR-transforming growth factor- $\alpha$ (TGF- $\alpha$ ) complex, and Yokoyama and colleagues looked at the EGFR-EGF complex. It was known that ligand-mediated EGFR dimerization occurs, but unlike other growth factor-receptor complexes, the researchers found that the ligand binds exclusively to a single EGFR molecule through specific regions of the EGFR extracellular domain that are outside the dimer interface. Instead of participating directly in the dimerization of receptors, the ligand seems to induce a conformational change in EGFR that allows a 'protruding arm' from each of the two receptors to then mediate dimerization and activation.

The findings have revealed a novel mechanism of receptor dimerization, and this understanding of EGFR activation at the three-dimensional structural level could provide a way to rationally design anticancer drugs that block signalling through inhibition of dimerization.

(1) References and links

Simon Frantz

ORIGINAL RESEARCH PAPERS Garrett, T. P. J. et al. Crystal structure of a truncated epidermal growth factor receptor extracellular domain bound to transforming growth factor- $\alpha$. Cell 110, 763-773 (2002) | Ogiso, H. et al. Crystal structure of the complex of human epidermal growth factor and receptor extracellular domains. Cel/ 110, 775-787 (2002) FURTHER READING Schlessinger, $\downarrow$

Ligand-induced, receptor-mediated dimerization and activation of EGF receptor. Cell 110, 669-672 (2002) 